\title{
LOS DESAFÍOS DE LA INDUSTRIALIZACIÓN: DEBATES Y PROPUESTAS PARLAMENTARIAS EN TORNO A LA EDUCACIÓN TÉCNICA INDUSTRIAL. CHILE 1929-1952*
}

\section{Francisco Rivera ${ }^{1}$}

\begin{abstract}
RESUMEN
El presente artículo tiene por objetivo analizar algunas propuestas referidas a la educación técnica industrial presentes en los debates de la Cámara de Diputados de Chile, en el contexto de las reformas económico-sociales derivadas, especialmente, de la crisis de 1929. En este sentido, se examinan las ideas y propuestas relacionadas con la formación de mano de obra calificada con el objetivo de salvar una de las principales limitaciones para el desarrollo de la modernización económico-social. Las transformaciones al sistema de educación técnica alcanzaron su mayor expresión en 1947, con la fundación de la Universidad Técnica del Estado, institución de nivel superior concebida como la encargada de formar a los profesionales orientados a sustentar los procesos de industrialización nacional.
\end{abstract}

Palabras clave: modernización, desarrollo industrial, educación técnica, Parlamento

\section{CHALLENGES OF INDUSTRIALIZATION: DEBATES AND PARLAMENTARY PROPOSALS AROUND INDUSTRIAL TECHNICAL EDUCATION. CHILE 1929-1952}

\begin{abstract}
This article analyzes some proposals related to the industrial technical education debates held in the Chamber of Deputies of Chile, in the context of economic and social reforms derived primarily from the 1929 crisis. In that sense, we examine ideas and proposals concerning to the formation of skilled labour to meet one of the major challenges to the socioeconomic modernization. The process of transformation of the technical education system reached its greatest expression with the founding of the Universidad Técnica del Estado (State Technical University) in 1947 conceived as the institution in charge of forming senior technical staff to support the implementation of national industrialization.
\end{abstract}

Keywords: modernization, industrial development, technical education, Parliament

* Este artículo se enmarca en el desarrollo del proyecto de investigación titulado: Educación Superior Técnica y Desarrollo Industrial: Ideas y Precursores. Chile 1930-1955, financiado por el Consejo Nacional de Educación (CNED), entre los meses de agosto de 2010 y abril de 2011. El autor agradece al CNED, al Decanato de la Facultad de Humanidades de la USACH por el patrocinio académico, y especialmente a Catalina Saldaña Lagos por la pesquisa de fuentes y comentarios críticos.

1 Facultad de Humanidades, Departamento de Historia, Universidad de Santiago de Chile, Santiago, Chile. Contacto: franciscoriverat@hotmail.com 


\section{LOS DESAFÍOS DE LA INDUSTRIALIZACIÓN: DEBATES Y PROPUESTAS PARLAMENTARIAS EN TORNO A LA EDUCACIÓN TÉCNICA INDUSTRIAL. CHILE 1929-1952}

\section{El primer tercio del siglo XX: nuevas ideas para viejos problemas}

La Primera Guerra Mundial puede considerarse el impulso inicial para las transformaciones que modificaron, al menos parcialmente, la estructura económica de Chile. Junto con inaugurar el siglo XX, el conflicto bélico abrió un tiempo histórico caracterizado, entre otros elementos, por el surgimiento de una serie de propuestas económicas y educativas. Las primeras relacionadas con el fomento y desarrollo de las industrias locales, mientras que las de tipo educacional con la formación de mano de obra calificada, necesaria para sustentar el proyecto de industrialización (Jobet, 1950; Lagos, 1960; Ortega 2005 , Meller, 2007)2. Durante la década de 1920, estas ideas de reforma económico-educativas se mantuvieron circunscritas especialmente a un restringido ámbito de ingenieros y técnicos, posición que fueron abandonando solo tras la Gran Depresión iniciada en el 29, al ser consideradas como las principales correctoras de las deficiencias del modelo primario exportador. El progresivo avance de estas ideas tendientes hacia la mayor participación del Estado y el fomento industrial, redundó en que hacia el último tercio de los años 30 fueran asumidas como proyecto de desarrollo nacional, siendo ejecutadas durante el transcurso de la Segunda Guerra Mundial. En general, las medidas económicas pasaron por instalar elementos reguladores al laissez faire predominante en la relación entre oferta y demanda, reconociéndose al Estado como un agente económico con atribuciones de planificación y fomento de los procesos de industrialización. Además, se adosó al Estado la función de facilitador de las inversiones

2 Cabe destacar que el proceso de industrialización se estaba lenta y paulatinamente desarrollando en Chile, al menos desde 1860, siendo catalizado por el contexto de posguerra. 
de los empresarios, a la vez que garante de ellas, lo que se expresó, por ejemplo, mediante la fundación de instituciones que propiciaron favorables condiciones crediticias para el empresariado nacional durante el gobierno de Carlos Ibáñez del Campo (1927-1931), como fueron los casos de la Caja de Crédito y Fomento Minero (CACREMI) ${ }^{3}$ en 1927 y el Instituto de Crédito Industrial en 1928, medidas que fueron reforzadas en 1939 con la creación de la Corporación de Fomento de la Producción (CORFO) (Ortega et al., 1989), bajo el gobierno de Pedro Aguirre Cerda (1938-1941), primer presidente de los gobiernos radicales que, como es sabido, asumieron el proyecto de fomento industrial. En consecuencia, entre la culminación de la Primera Guerra en 1919 y el fin de la Segunda en 1945, crisis del 29 mediante, las ideas referidas al desarrollo de la industria y a la educación técnica como sustento de ésta, pasaron de una condición de marginalidad a ser ejecutadas por el aparato Estatal, constituyéndose en transformaciones globales que remecieron sus estructuras económicas a través de la implementación del modelo de Industrialización Sustitutiva de Importaciones (ISI) (Devés, 2000, 2003, 2004).

Sin embargo, en este marco de ideas donde Estado e industrialización aparecían como las variables para conjugar, y a pesar del consenso en relación con esta primicia por parte de los diversos actores sociales o colectivos que elaboraron las propuestas, deben reconocerse múltiples divergencias respecto de la implementación de este proyecto de modernización económico-social. Estos matices se produjeron en relación a las estrategias para conquistar dicho proyecto, proceso en el que se realizaron diversos diagnósticos respecto a las limitaciones para implementar las propuestas. Una de las principales radicó en la falta de cuadros técnicos capacitados para conducir el proceso de industrialización, lo que obligaba a generar diversas reformas en el sistema de educación técnica nacional. En este sentido, el Congreso constituyó un espacio de debate en el que se sintetizaron las ideas y proyectos referidos a optar por el camino de la industrialización, salvando las limitaciones antes señaladas.

3 Cabe destacar que la CACREMI se originó en estudios realizados por la Sociedad Nacional de Minería (SONAMI), que elaboró el proyecto de ley. Esto releva el rol de los empresarios en el diseño de políticas de fomento crediticio para el desarrollo de la minería nacional. 
El presente artículo tiene por objetivo examinar las ideas y propuestas relacionadas con el fomento de la educación técnica industrial, originadas en las discusiones sostenidas en la Cámara de Diputados y en el Senado, que acompañaron los debates orientados al impulso y ejecución del modelo ISI como política de modernización económica, entre los años 1929 y 1952. En la Cámara se expresaron las orientaciones de diversos partidos políticos, a la vez que pueden concebirse como una síntesis de las demandas que realizaron otros actores sociales, como por ejemplo los estudiantes de escuelas técnicas, cuyas demandas se recogen y exponen en el periodo estudiado. De este modo, consideramos que las propuestas referidas a la educación técnica, como soporte de los procesos de industrialización, no solo constituyen extensiones de las plataformas políticas de los partidos que representaron cada uno de los parlamentarios, sino que también de otros grupos que, a través de las discusiones, se consolidaron como interlocutores en los debates referidos a la educación técnica. Cabe destacar que, a partir de la revisión de los diarios de sesiones, como fuentes pertinentes para la investigación, se puede concluir que el grueso de las propuestas provino desde la Cámara de Diputados y, en muy menor medida desde el Senado, que actuó como simple sancionador, o caja de resonancia, de los argumentos esgrimidos por la Cámara baja.

A partir de los debates y propuestas analizados, sostenemos como tesis general que en el periodo estudiado existió consenso en torno a la necesidad de fomentar la industrialización de la producción, estimulando la consiguiente reforma al sistema de educación técnica como medio para sustentar dicho proceso. A su vez, y a pesar de los matices, la idea entre los diversos actores políticos de que dichas reformas debían ser conducidas y ejecutadas por operarios chilenos dotó al proceso de un carácter nacional. Cabe destacar que, tanto en el ámbito económico como en el educativo, las propuestas surgieron al unísono de la crisis de 1929, o incluso con un par de años de anterioridad a ella. Sin embargo, a medida que avanzaban los efectos de la depresión ambas variables se fueron disociando en los tiempos de implementación, oponiéndose a la velocidad de las reformas económicas, catalizadas con la fundación de la CORFO en el 39. La lentitud de las transformaciones educativas, que solo en 1952 tomaron 
forma con la promulgación del Estatuto Orgánico de la Universidad Técnica del Estado (UTE), institución que no se pudo ocupar en todo su potencial, puesto que comenzó sus labores en la antesala de la crisis que afectó al modelo de Industrialización Sustitutiva de Importaciones hacia mediados de los 50. En síntesis, entre los años 1929-1952, junto con la primacía de lo nacional en el discurso de los políticos, tanto las reformas económicas como las educativas se distinguieron por su carácter parcial y disociación en los tiempos de implementación. Las reformas económicas emanadas de un contexto de inestabilidad económico-social es lo que analizamos a continuación.

\section{El problema industrial: la crisis de 1929 y el cuestionamiento al modelo primario exportador}

El primer elemento presente en las discusiones parlamentarias al momento de la crisis del 29 radica en el diagnóstico de que el modelo de economía primario exportadora imperante en Chile había fracasado, por lo que debía sufrir transformaciones tendientes a corregir la alta vulnerabilidad derivada de su dependencia a la demanda externa, lo que, al menos, tuvo expresión continental. En este análisis, la Primera Guerra Mundial fue concebida como la antesala, o más bien, la génesis de la crisis que a la sazón golpeaba a la economía nacional. El conflicto fue caracterizado como el causante de "todas nuestras estrecheces financieras" (Cámara de Diputados (CD), Diario de Sesiones (Ss.) 8a ordinaria, 10 de junio de 1931, p. 223.) que generaron el caos económico, evaluación compartida por los representes de las distintas bancadas políticas.

Los cuestionamientos a la estructura económica nacional estuvieron acompañados de una fuerte crítica a las autoridades responsables, acusadas de no tomar precauciones en los tiempos de bonanza del salitre, ni menos luego de la advertencia al modelo primario exportador que había significado la Primera Guerra, situación que dificultaba aún más la realidad económica tras la crisis del 29. En este contexto, las opiniones vertidas en junio de 1931 por el diputado del Partido Demócrata, Alejandro Cataldo Maureira respecto de la producción del nitrato, son categóricas, señalando que Chile antes de la guerra tenía un espléndido mercado salitrero, pero que: 
"no hubo previsión por parte de nuestros productores de nitrato para estudiar los mercados, hacer cálculos para estandarizar los precios y la producción, de rebajar el costo de elaboración. No hubo, [...] sino el despilfarro más estrepitoso en manos de capitalistas extranjeros de toda una riqueza nuestra, de una riqueza que debió ser patrimonio nacional, de una riqueza que hoy solo existe en los cascarones de la pampa, que es el cementerio del trabajo, donde ni siquiera hubo ley para los pobres rotos que la habían conquistado" (CD, Ss. 8 a ordinaria, 10 de junio de 1931, p. 224.)

Si la guerra europea había inaugurado "una serie de nuevos problemas políticos, sociales y económicos, que al encararlos, han cambiado totalmente la estructura de los Estados" (CD, Ss. $8^{\text {a }}$ ordinaria, 10 de junio de 1931, p. 223.), la crisis del 29, los había profundizado. La falta de previsión era considerada la principal causa de la decadencia de trabajo, que azotaba "con marcada crueldad a la porción más considerable de nuestros conciudadanos" (CD, Ss. $8^{\text {a }}$ ordinaria, 10 de junio de 1931, p. 222.). El aspecto social, resultaba para los parlamentarios tanto o más dramático que el económico; un muy citado informe de la Liga de las Naciones, titulado Word Economic Survey (1923-1933), señaló que Chile fue el país más golpeado por la Gran Depresión (Aylwin, 2002). En 1930, el diputado del Partido Demócrata Pedro Pablo Navarrete daba las primeras luces de estos efectos, al señalar que:

"las diferentes industrias fabriles pasan por una crisis aguda, que está alarmando a los industriales, en especial a los obreros, que ya sufren la miseria con sus funestas consecuencias por falta de trabajo. Y esto es muy doloroso si se considera el mérito de hombres, que han dedicado los mejores años de su vida para adquirir los conocimientos que los capacite para ganarse la vida honradamente" (CD, Ss. $12^{\text {a }}$ extraordinaria, 9 de diciembre de 1930 , p. 525.)

Frente a este escenario económico-social, comenzaron a surgir desde el hemiciclo una serie de propuestas de carácter programáticoproyectual, todas ellas orientadas a superar la crisis económica y 
las condiciones de precariedad material de la población. Dichas propuestas complementaron las primeras medidas del gobierno, que se destinaron a la ejecución de grandes obras públicas para dotar de empleo temporal a los sectores más afectados, a fin de evitar la desocupación y sus funestas consecuencias ${ }^{4}$. Como señalan varios autores, en la época primó la idea de imitar la ruta seguida por las economías centrales, donde el mejoramiento de las condiciones de vida de la población se encontraba estrechamente vinculado al desarrollo de la industria local, vale decir, el progreso social dependía del económico y viceversa, constituyendo un antecedente de lo que en las décadas del 40 y 50 fue conocido como el pensamiento o paradigma desarrollista-modernizador (Aguirre Cerda, 1933; Jobet, 1955; Pinto, 1959; Muñoz 1986; Fáundez, 1992; Devés 2000; Moulian, 2006; Milos, 2008).

El representante demócrata Alejandro Cataldo, en su intervención de 1931, fue enfático al señalar que "el porvenir de nuestros países está en el fomento industrial, en el estímulo a las industrias para fortalecer nuestra capacidad productora y poder competir en otros mercados" (CD, Ss. $8^{a}$ ordinaria, 10 de junio de 1931, p. 226). A juicio del parlamentario, en la búsqueda por la expansión del radio financiero del país, se hacía fundamental la urgente intervención del Estado en las industrias de su propiedad, y especialmente en las particulares, pero "en forma que contemple con justicia los intereses colectivos" (CD, Ss. $8^{\text {a }}$ ordinaria, 10 de junio de 1931, p. 227). El diputado argumentaba que el Estado chileno podía procurarse cuantiosas entradas, "controlando la producción, fijando el costo, la venta, y por ende, limitando las utilidades del capital. Y precisamente con castigo al sobreprecio de la elaboración, que en este país está al capricho de los capitalistas" (CD, Ss. $8^{a}$ ordinaria, 10 de junio de 1931, p. 226). Las medidas a favor del otorgamiento de un rol preponderante a la intervención estatal generadas a principios de 1930, mantuvieron su consenso, al menos por dos décadas, no solo entre aquellos parlamentarios proclives a las ideas de izquierda sino también a los de derecha, quienes tenían como modelo las medidas

4 En su intervención del 27 de agosto de 1930, Pedro Navarrete profundiza en las medidas tomadas por el gobierno y la condición de los sectores populares. Ver CD, Ss. $42^{a}$ ordinaria 27 , p. 1995 y ss. 
proteccionistas tomadas por Estados Unidos, especialmente, respecto a la dictación, en 1933, en el gobierno de Franklin Delano Roosevelt, de la ley de resurgimiento industrial conocida como NIRA, que puso énfasis en la recuperación de la industria, pero también en la cooperación entre los grupos industriales, en mejorar las condiciones de trabajo como asimismo de mantener y garantizar la unidad de acción entre trabajadores y empresarios ${ }^{5}$. En Chile, las propuestas de los parlamentarios se orientaron además a cambiar la mentalidad de este último grupo, pues se consideraba que algunos empresarios se habían aprovechado de la crisis económica especulando y despidiendo injustificadamente a trabajadores. En 1930, el diputado de la Confederación Republicana de Acción Cívica Luis Moreno Fontanez alertó de esta situación, señalando que en el contexto de crisis industrial no existía por parte del Estado un control "para poder establecer la veracidad de las afirmaciones de estos industriales que se quejan de la crisis, [por lo que] de la noche a la mañana los obreros se encuentran que quedan sin trabajo" (CD, Ss. 50 a ordinaria, 10 de septiembre de 1930, p. 2387).

La intervención del Estado se planteaba no solo respecto de la administración y regulación, sino que principalmente para el fomento de la industrialización o de lo que fue definido como 'capacidad industriosa' del país, concebida como la palanca que pondría término "a lo que somos hasta la fecha: una factoría de mercados extranjeros y esclavos de la economía internacional" (CD, Ss. $24^{\text {a }}$ ordinaria, 20 de julio de 1931, p. 941). En los debates parlamentarios, sistemáticamente se hace hincapié en la amplia presencia de recursos naturales existentes en Chile, todos ellos potencialmente explotables a escala industrial con la finalidad de promover su inserción en los mercados externos. La intervención de Rudecindo Ortega Masson, diputado del Partido Radical Doctrinario, en su intervención del 14 de julio de 1931, expuso estos elementos, al señalar que

"el Gobierno debe desarrollar iniciativas encaminadas a la creación de nuevas industrias en el país, debe pensar

5 Al respecto, se recomienda analizar las discusiones de la CD, Ss. $35^{\text {a }}$ ordinaria, del 25 de julio de 1945, pp. 1291-1293, donde se plantea el reconocimiento del modelo estadounidense y su recomendación para el caso chileno. 
seriamente en entonar nuestra vida económica con actividades que, a la vez que representen producción de materias primas, abran nuevos mercados de consumo, que se traducirían en trabajo y bienestar para el país" (CD, Ss. $22^{\mathrm{a}}$ ordinaria 14 julio de 1931, p. 863).

Si bien hubo consenso en enfatizar la industrialización y diversificación de los productos destinados a la exportación como medidas orientadas a disminuir las fluctuaciones económicas sufridas por el país, las discusiones parlamentarias dan cuenta de matices en torno a la definición de industria o más bien de qué industria potenciar. En este sentido, primó en los debates la idea de consolidar las industrias para las cuales se emplearan materias primas presentes en el país -principalmente las orientadas a la producción de alimentos- antes que de aquellas consideradas 'pesadas', vinculadas entre otros elementos con producción de energía, el hierro y la petroquímica, las que requerían de mayor sofisticación tecnológica. Ciertamente, optar por la industria liviana constituía un paliativo orientado a mejorar las condiciones de la economía nacional frente a las fluctuaciones externas, mas no la sacaría de su posición periférica en las relaciones económicas internacionales, situación propia de las economías primario exportadoras. Desde inicios de la década de 1930 estas medidas, por muy parciales que fueran, eran compartidas incluso por aquellos diputados que abogaban por el desarrollo de la industria pesada, pues eran conscientes de las limitaciones previas que se debían salvar. Ello no significó -en todos los casos- renunciar al proyecto de fomento a la industria para la independencia económica sino que se impuso la opción por avanzar paulatinamente en este sentido. En este objetivo se enmarcan las diversas medidas orientadas a reforzar la función del Estado como regulador, inversor y facilitador de la inversión privada para el fomento de la industria nacional, que alcanzaron su cenit con la creación de la CORFO en $1939^{6}$, además de las reformas al sistema de educación técnica, definidas como pilares del modelo ISI.

6 La CORFO fue definida por el diputado del Partido Radical, Hermes Ahumada Pacheco, como "una de las herramientas más poderosas para intensificar la producción, y, en parte, nuestra economía hacia una industrialización progresiva [...] creada gracias al espíritu inmortal del presidente Pedro Aguirre Cerda". Ver, Ss. 18ª ordinaria, 27 de junio de 1945 , p. 583 y ss. 
Otra de las limitaciones principales, además de las mencionadas con anterioridad, radicó en la incapacidad de la mano de obra nacional para cumplir con las labores industriales más complejas. El diputado Pedro Navarrete, en un contexto donde se hacían sentir los primeros efectos de la crisis económica, ofreció un interesante diagnóstico y acertada síntesis de la condición de las industrias nacionales con relación a sus capacidades productivas y mano de obra, señalando en 1930 que:

"hay unas que con poco dinero y reducidos operarios proporcionan grandes rendimientos; otras que requieren fuertes capitales pero no necesitan de obreros preparados, sino de operarios de poco costo; otras que no solo requieren capitales cuantiosos sino que, para su buena marcha, exigen operarios técnicos cuyo sostenimiento consume la mayor parte de sus utilidades" (CD, Ss. 42a ordinaria, 20 de agosto de 1930, p. 1997).

Junto a lo contingente del diagnóstico realizado por el diputado, se debe destacar la temprana conciencia que existió entre los parlamentarios de que una de las principales limitaciones para el impulso industrial era la insuficiente capacitación de la mano de obra. El mismo año 29 el diputado Rogelio Ugarte planteó que:

"solo nos falta el factor humano, el obrero competente, amante de su oficio, honrado en el trabajo y sobrio en la vida; y ese elemento primario es el que debemos formar, sacándolo desde la infancia propicia para la modelación de los espíritus, para fortalecer y adiestrar el músculo que haya de moverse con inteligencia y con alma en la obra perfecta" (CD, Ss. $24^{a}$ ordinaria, 22 de julio de 1929, p. 999).

Lejos de disminuir con el correr de los meses este diagnóstico aumentó, sumándose a él las críticas en contra del énfasis puesto en la educación humanístico-científica que habían tenido las reformas educacionales, en oposición de las acontecidas en la educación de tipo técnico-vocacional, lo que era evaluado como una de las principales causas de la postración económica del país. En esta línea argumentativa se encuentra la opinión vertida en 1931 por el diputado Rudecindo Ortega, al sostener que 
"nos encontramos hoy con que después de cuatro años de sucesivas reformas, de este verdadero vendaval de innovaciones que ha azotado a nuestras instituciones docentes, el enfermo está con su salud más quebrada que antes. [...] frente al problema más grave del momento, que es el problema económico, vale la pena que recordemos lo que se olvidó al considerar la cuestión educacional" (CD, Ss. 22a ordinaria, 14 de julio de 1931, pp. 860-861).

En consecuencia, el anhelo de dar vida a una nueva fase de industrialización topaba con la incapacidad del sistema educativo chileno. En este escenario, se presentaron para la discusión parlamentaria diversos proyectos orientados a reformar la educación técnica, como plataforma para el impulso industrial, tema al que nos abocamos en el siguiente apartado.

\section{El problema educativo: propuestas de reforma a la enseñanza técnica industrial}

\section{a. La Universidad Municipal del Trabajo y el Instituto de Perfeccionamiento Técnico}

De forma paralela a las situaciones referidas anteriormente, se planteó en el Congreso la urgente necesidad de reformular el sistema de educación técnica, evaluado como insuficiente para cumplir con las nuevas demandas de desarrollo de la economía nacional. Por este motivo, progresivamente las propuestas avanzaron desde el mejoramiento y ampliación de la estructura educativa existente a la idea de corregir las profundas deficiencias del sistema educativo, mediante la fundación de una institución de educación superior encargada de formar cuadros técnicos capaces de sustentar los procesos de industrialización. Estas disposiciones se orientaron además hacia el mejoramiento de las condiciones materiales de la población mediante su mayor calificación para los procesos productivos. Como ya señalamos, en la coyuntura de la crisis del 29 este último aspecto, vinculado con la marginalidad económica de gran parte de la sociedad y especialmente de los sectores populares, cobró urgencia. Las propuestas de reforma de la enseñanza técnica 
surgieron de la Cámara de Diputados, apelando a una experiencia de instrucción anterior y como una medida inmediata destinada a mejorar el acceso a la formación técnica de los sectores más desposeídos. Así, se planteó la idea de revivir las funciones realizadas por la Universidad Municipal del Trabajo (UMT) a principios de los años 20.

El proyecto de refundar la Universidad fue presentado por el diputado del Partido Comunista (PC) Abraham Quevedo Vega en julio de 1929. Este diputado orientaba sus esfuerzos a restablecer lo que originalmente fue la Universidad del Trabajo, creada por la Municipalidad de Santiago en el año 1923, a semejanza de las universidades de este tipo existentes en Estados Unidos y en casi todos los países europeos, especialmente a la UMT de la ciudad de Charleroi, en Bélgica ${ }^{7}$. En Chile, el militante del Partido Radical (PR), Rogelio Ugarte Bustamante fue el encargado de fundarla en su calidad de primer alcalde de la ciudad de Santiago ${ }^{8}$. Una editorial del periódico El Mercurio fechada 17 de diciembre de 1923 caracterizó la obra y función de la UMT:

"La Universidad del Trabajo tiene por objeto educar a los obreros y obreras en las artes y oficios que les sirvan para ganarse la vida, perfeccionando sus conocimientos técnicos, al mismo tiempo que su práctica manual, a fin de que la obra resulte tan perfecta como es posible dentro de las condiciones de inteligencia y perseverancia del individuo. Esta enseñanza técnica, difundida entre los obreros, es la base de todo progreso industrial" ( $E l$ Mercurio, 17 de diciembre de 1923).

Apelando a ejemplos pretéritos, el diputado Quevedo buscaba resolver no solo la falta de calificación de la mano de obra, importante obstáculo para el naciente proceso de industrialización, sino también elevar las condiciones materiales de los sectores populares, en el entendido de que los efectos más dramáticos de la crisis económica, en

7 Al respecto de las influencias recogidas para la fundación en 1923 de la UMT de Santiago, se recomienda leer la intervención del diputado Abraham Quevedo Vega en la Ss. $23^{a}$ ordinaria, del 17 de julio 1929, p. 957 y ss.

8 Entre los años 1900-1924, por la Ley de Comuna Autónoma, existían tres alcaldes por Comuna. El militante del Partido Radical, Rogelio Ugarte Bustamante, fue alcalde de Santiago entre el 4 de abril de 1923 y el 4 de mayo de 1924. 
el mes de julio de 1929, aún no azotaban con su mayor fuerza a dicho grupo social. Por estos motivos, precisamente, el carácter integrador con el que se había fundado la universidad original era mantenido en la propuesta de Quevedo, asumiendo las palabras sostenidas por Rogelio Ugarte Bustamante, en 1923, en las que el diputado radical afirmaba que:

"la acción de la Universidad alcanzará, pues, a todo el que se interese por progresar, sin distinción de clase, sexo, edad u ocupación. Sus aulas y talleres estarán de par en par abiertas, de día y de noche, para el niño que, habiendo cumplido con su obligación escolar primaria, esté en condiciones de dedicar todo su tiempo al estudio y al trabajo; para el obrero que se resuelve a robar algunas horas a su descanso, para dedicarlas a su perfeccionamiento industrial y a aumentar así su capacidad productora; [...] para el que sólo disponga de un día a la semana o de una hora en cada día y desee dedicar ese tiempo a aumentar su capacidad productiva.

Será, pues, la Universidad Municipal del Trabajo que se acaba de fundar, una institución que habrá de contribuir poderosamente a nuestra mayor cultura, a la formación de la futura masa industrial del país y al incremento de la riqueza nacional" (CD, Ss $23^{\text {a }}$ sesión ordinaria 17 de julio de 1929, p. 957).

Otra editorial, esta vez de El Diario Ilustrado, publicada el 18 de diciembre de 1923, es pertinente para caracterizar a los estudiantes de la Universidad Municipal del Trabajo. Según el periódico, la institución "es una escuela profesional o de artes y oficios con dos grandes secciones: una para hombres y otra para mujeres" (El Diario Ilustrado 18 de diciembre de 1923) Además, permite profundizar en las funciones y oficios impartidos en la Universidad, entre las cuales, "la carpintería es sin duda alguna, la madre de las industrias masculinas, [y] las labores de aguja el resumen de las femeninas"(CD, Ss. $23^{a}$ ordinaria 17 de julio de 1929 , p. 959). Cabe señalar que la aspiración de este modelo educativo radicaba en conseguir que alrededor de las funciones antes señaladas, se agruparán otras que 
superaran el campo del ejercicio manual, orientándose a la educación de la mujer para labores propias del hogar, y al hombre para realizar "una serie de pequeñas labores que a diario solicitan su actividad en la vida corriente" (CD, Ss. 23a ordinaria 17 de julio de 1929, p. 959.), vale decir, a generar una división social de las actividades, con la consiguiente moralización de los y las estudiantes. En su propuesta, el diputado Quevedo realizó dichos objetivos al enfatizar que:

"es necesario que el gañán y al simple ganador del mendrugo diario, que se debate en medio de la miseria, de la ignorancia y de los vicios, suceda el ciudadano compresor de su alto y noble rol, como obrero de conciencia y jefe de hogar sencillo que merece nuestra patria [...]. Dignifiquemos el trabajo manual, formemos el templo para su culto, construyamos la Universidad del Trabajo, así, con ese nombre que no es inapropiado ni pomposo, y habremos iniciado la propulsión más soberbia para aplastar muchos males sociales y preparar mejores destinos a nuestro pueblo" (CD, Ss. 24 a sesión, 22 de julio de 1929, p. 999).

La UMT se conformaba de un conjunto de escuelas-talleres donde solo se impartía docencia técnica en niveles básicos y medios, constituyendo más un esfuerzo por modelar conductas y dotar de conocimiento prácticos a los sectores populares, antes de corresponder a una universidad en la concepción de 'universidad compleja', vale decir, que cumpliera funciones superiores a la docencia, desarrollando investigación y extensión. Del mismo modo, a pesar de parecer su título un tanto ampuloso, lo que se buscaba con él era también dignificar el trabajo manual, destinando a su desarrollo un organismo racionalmente organizado, que contrarrestara además la apreciación $\mathrm{y}$ trato peyorativo de quienes optaban por el examen del bachillerato o ejercían profesiones liberales ${ }^{9}$. En suma, la idea de revivir la UMT se orientó a cumplir una doble función: ser una manera práctica

9 Al respecto, es pertinente citar la editorial de El Diario Ilustrado del 18 de diciembre de 1923. También sesiones Cámara de Diputados, año 1929, p. 969, correspondiente a la 23ª Sesión Ordinaria, realizada el 17 de julio, donde el bachillerato es considerado como una 'plaga', señalándose que "no todo ha de ser ciencia, literatura, leyes, medicina, carreras liberales. El trabajo merece los honores de un instituto especial [...] la Universidad Municipal del Trabajo", p. 969. 
de solucionar el problema de la preparación técnica de los obreros nacionales a la vez que constituirse en un primer impulso al desarrollo del trabajo manual.

Como puede desprenderse de las palabras del diputado Quevedo, la urgencia por fundar planteles destinados a la educación técnica y los esfuerzos por desarrollar procesos formativos de mano de obra calificada de nivel superior, que se intensificarán en la década de 1940, se relacionaron no solo con el aspecto económico, en tanto generación de obreros capacitados en los procesos productivos industriales, sino también con la moralización y corrección de aquellas conductas evaluadas como nocivas para el progreso de la nación, promoviendo la generación de nuevos hábitos en los individuos. En consecuencia, consideramos que sería insuficiente analizar las ideas orientadas a la conquista del progreso unicamente desde el aspecto económico, en tanto la suma de procesos formativos de mano de obra calificada, entendida como simple factor productivo, sino que este proceso contó también con una arista social, vinculada a la reconversión de las conductas de los sectores populares mediante una formación que les imprimiera una 'moral' productiva nueva. Así, y como tantas veces en la historia nacional, progreso y 'civilización' asomaron nuevamente de la mano.

El proyecto del diputado Quevedo Vega buscaba complejizar la UMT, concibiéndola como un componente importante de un programa integral de reforma del sistema de educación técnica. Esta intención la expresó en la 24ª Sesión Ordinaria del 22 de julio de 1929, donde invitaba a que el Presidente de la República tomara la UMT "como una hija adoptiva y darle vida con el calor de sus ansias patrióticas" (CD, Ss. 24a ordinaria, 22 de julio de 1929, p. 999).

A su vez, esta primera experiencia vinculada a la reforma de la educación técnica venía además a completar la reforma del sistema educativo científico-humanista iniciada con la aprobación de la Ley de Instrucción Primaria Obligatoria en 1920. Nueve años después de su aprobación, la Ley ya daba sus primeros frutos, por lo que se estimaba que en el nuevo escenario económico y social el problema no era tanto "enseñar a leer a los analfabetos, sino preparar a los obreros 
de mañana, de modo de capacitarlos para el ejercicio de todos los oficios y preparar así la futura masa industrial del país" (CD, Ss. $23^{\text {a }}$ ordinaria, 17 de julio de 1929, p. 957).

Si bien la idea de reflotar o ampliar las funciones que había cumplido la UMT no llegó a su ejecución, el problema de la ampliación y complejización del sistema de educación técnica continuó presente en los debates parlamentarios. En esta línea se fueron sucediendo los proyectos destinados a su transformación. Durante agosto de 1934, el diputado ${ }^{10}$ Prudencio Garrido Salazar presentó un proyecto destinado a fundar un Instituto de Perfeccionamiento de los Trabajadores de Chile, el que fue cotejado con los ministros de Instrucción y Trabajo, constituyendo un ejemplo de esta complejización en ciernes. En su argumentación, el parlamentario sostuvo que a la sazón el sistema de educación técnica se hacía insuficiente, puesto que se había comprobado en los hechos el problema de la industria nacional, que a pesar de ser incipiente todavía, necesitaba de un "mayor número de técnicos hábiles y capaces para desarrollarse" (CD, Ss. 46ª ordinaria, 27 de agosto de 1934, p. 2476.), pues a su juicio:

"Los obreros con que actualmente cuenta, si bien es cierto, inteligentes y esforzados, carecen de la técnica o teoría de las profesiones; sus conocimientos han sido adquiridos en el taller, junto a la fragua, frente al yunque, pero no en la escuela, que es la única que da conocimientos técnicos. [...]. De aquí la necesidad de que nuestros asalariados en general cuenten con medios de perfeccionar sus conocimientos profesionales y logren mediante una educación adecuada los que deben aplicar en su desempeño profesional. Las escuelas dominicales y nocturnas que actualmente existen satisfacen en parte esa función" (CD, Ss. 46 ordinaria, 27 de agosto de 1934, p. 2476).

En cuanto al funcionamiento, el Instituto de Perfeccionamiento Técnico o Instituto de los Trabajadores de Chile sería una corporación

10 Prudencio Garrido Salazar, fue electo diputado en 1930 como Confederación Republicana de Acción Cívica de Obreros y Empleados de Chile (CRAC), entidad de tendencia de izquierda, fundada en diciembre de 1929 y disuelta a la caída de Ibáñez 1931, por lo que al momento de la presentación de su propuesta, Garrido tenía carácter de independiente. 
privada, financiada por el Estado mediante un aporte en dinero y el otorgamiento de franquicias por un número limitado de años, que no fueron definidos en el proyecto. No obstante, una vez que el Instituto "hubiese capitalizado lo suficiente seguiría como corporación privada bajo la custodia fiscal, en lo que respecta a su misión educadora" (CD, Ss. 46ª ordinaria, 27 de agosto 1934, p 2477-2478.), pero su financiamiento se haría entonces por medio del pago de aranceles por parte de los propios alumnos. El Instituto dependería del Ministerio del Trabajo y basaría la preparación profesional de los empleados y obreros "mediante el uso del sistema de enseñanza por correspondencia y el empleo de todos los métodos activos para perfeccionar la capacitación profesional de los trabajadores" (CD, Ss. 46a , ordinaria 27 de agosto de 1934, p. 2478). De esta forma, el taller daría a los obreros/estudiantes la práctica y la escuela, por correspondencia, los conocimientos teóricos de su profesión. Esto último entregaba un cariz innovador, acorde con las modernizaciones de los procesos productivos.

\section{b. La crisis de la Madre Escuela: las limitaciones de la Escuela de Artes y Oficios (EAO)}

En los proyectos de reforma al sistema de educación técnica nacional presentados por los parlamentarios se estableció paulatinamente la necesidad de fundar una institución superior de carácter más complejo que las antes reseñadas, que además de soportar la industrialización, satisficiera la creciente demanda por incorporarse a la educación técnica. Según el diputado Prudencio Garrido, hacia el año 1934 "las escuelas profesionales [se encontraban] llenas de alumnos y la EAO ha debido duplicar su capacidad" (CD, Ss. 23 $3^{\text {a }}$ ordinaria, 17 de julio de 1929, p. 958). Fundada en 1849, la Escuela de Artes y Oficios constituía la primera escuela técnica chilena y hasta ese momento el principal espacio de formación de técnicos y artesanos a nivel nacional, por lo que las propuestas de reforma al sistema de educación técnica la instalaron en el centro del proyecto encauzado a potenciarla. La intención de complejizar la EAO fue expresada, entre otros, por el diputado Abraham Quevedo, para quien solo:

"bastaría con ampliar nuestra EAO y transformarla en lo que debe ser: la formadora de obreros de todas las artes 
manuales, la descubridora e impulsora de todos aquellos que tengan condiciones de artífices, y la seleccionadora y protectora de los más capaces" (CD, Ss. $24^{\text {a }}$ ordinaria, 22 de julio de 1929, p. 999).

De esta forma, el proyecto de Quevedo recurrió a una experiencia educativa del siglo XIX: la EAO, estableciéndola como un punto de partida para tomar el impulso hacia la satisfacción de los objetivos trazados. Sin embargo, dicha complejización orientada hacia el 'deber ser' de la Madre Escuela, topaba con graves problemas de funcionamiento interno y vínculo con el exterior, que propiciaron el diagnóstico de que la Escuela era incapaz -tal cual se encontraba a principios de los 30- de protagonizar la formación de cuadros calificados para conducir las transformaciones industriales.

En el año 1931, los estudiantes y exalumnos de la EAO iniciaron una serie de protestas en contra de la administración del ingeniero Ramón Montero, hermano del entonces Presidente de la República, Juan Esteban Montero (1931-1933), acusado de situarse en una posición de "aislamiento respecto a los alumnos" (CD, Ss. 49a ordinaria, 31 de agosto de 1931, p. 1795) que impedía el contacto fluido entre éstos y el director general, fracasando los esfuerzos por "realizar una obra verdaderamente educativa" (CD, Ss. 49ª ordinaria, 31 de agosto de 1931, p. 1795). Las demandas de los exalumnos fueron transmitidas en el hemiciclo por el diputado comunista Abraham Quevedo Vega en agosto de 1931; según el parlamentario los estudiantes:

"manifiestan que la enseñanza industrial que se da en esa Escuela ha fracasado por completo, se me afirma que los jóvenes salidos de ese establecimiento si han surgido en sus actividades no es por el aprendizaje que han tenido en ese establecimiento, no es por las enseñanzas de sus maestros, sino por los conocimientos adquiridos después en la lucha misma por la vida y en los estudios especiales a que se dedican pero que la gran mayoría de estos jóvenes fracasan por la deficiencia de la enseñanza" (CD, Ss. 49a ordinaria, 31 de agosto de 1931, p. 1801-1802). 
Como hemos señalado, las demandas y proyectos por la reforma de la educación técnica se incorporaron dentro de un proyecto mayor vinculado al desarrollo nacional. Esto a su vez provocó que el carácter nacionalista de su implementación fuera enfatizado tanto por los parlamentarios como por los estudiantes, situándolo como un elemento central en el diseño de la propuesta educativa. El énfasis de las críticas a la formación se encontraba puesto en dos aspectos férreamente vinculados: a) el hecho de que la formación de los operarios recayera en profesores extranjeros, y b) la contratación de mano de obra extranjera en la Escuela, evaluada como excesiva.

En su intervención, Abraham Quevedo puso de relieve las críticas de los estudiantes al proceso de formación, analizado como ineficiente debido, fundamentalmente, a factores como:

"lo anticuado de los programas y por la incapacidad de los maestros, principalmente de los maestros extranjeros que existen en ese establecimiento. La incapacidad de estos maestros, según estos ex alumnos, es la causa principal de su fracaso en las diversas actividades de la vida" (CD, Ss. 49ª ordinaria, 31 de agosto de 1931, p. 1801-1802).

En este contexto, las críticas de los estudiantes de la EAO giraron en torno a la función formativa encabezada por maestros extranjeros cuya incapacidad, según los exalumnos, constituía "la causa principal de su fracaso en las diversas actividades" (CD Ss. 49a ordinaria, 31 de agosto de 1931, p. 1801) y a las deficiencias de los programas de estudio, evaluados como necesarios de modificar, a fin de hacerlos "más de acuerdo con la realidad de la vida" (CD, Ss. 49ª ordinaria, 31 de agosto de 1931, p. 1795). Esto último era considerado un problema producido porque "los maestros extranjeros de la escuela no conocen nuestra idiosincrasia, ni los secretos para triunfar en la lucha por la existencia en el país" (CD, Ss. 49ª ordinaria, 31 de agosto de 1931, p. 1802). Esto traslucía la deficiencia de la Escuela de Artes y Oficios respecto de "muchos conocimientos técnicos y prácticos para poder formar verdaderos obreros" (CD, Ss. 49a ordinaria, 31 de agosto de 1931, p. 1804.). La solución a estos problemas pasaba para el diputado Navarrete por traer a la Escuela "técnicos alemanes que solo saben hacer 
una tuerca o un tornillo" (CD, Ss. 49a ordinaria, 31 de agosto de 1931, p. 1804) siendo que los obreros nacionales debían estar preparados para hacer toda clase de materiales dentro de los talleres.

La contratación de mano de obra extranjera constituyó el otro pilar de las demandas de los alumnos. El mismo diputado Quevedo fue el encargado de plantear las quejas de los estudiantes de la EAO respecto de que en su enseñanza existía cierto extranjerismo, principalmente alemán, "que va en contra de un racional nacionalismo que, según ellos, debe existir en la mencionada Escuela"(CD, Ss. 49a ordinaria, 31 de agosto de 1931, p. 1791). Para los alumnos:

"la obra de modernización que se ha hecho en la escuela con maquinaria alemana y la contratación de técnicos de la misma nacionalidad, equivale más bien a una germanización de la enseñanza, que ha provocado un desplazamiento del personal chileno" (CD, Ss. 49a ordinaria, 31 de agosto de 1931, p. 1797)

El diputado Prudencio Garrido arremetió denunciando que ante la situación de carencia de escuelas técnicas "nuestros obreros son desplazados de ordinario por técnicos extranjeros que ganan fabulosos sueldos y nada en común tienen con el sentimiento de nacionalidad" (CD, Ss. 49ª ordinaria, 31 de agosto de 1931, p. 1802). Situación que se agravaba en el entendido de que:

"casi todas las grandes industrias de este país están dirigidas por extranjeros, debiendo en mi concepto figurar como directores de estas mismas industrias los alumnos que salen de este establecimiento, que debería ser una verdadera universidad del trabajo" (CD, Ss. 49a ordinaria, 31 de agosto de 1931, p. 1802)

Así como Abraham Quevedo hizo suyas las demandas de los estudiantes en pro de los de origen nacional y en contra de la abundante presencia alemana, el diputado del Partido Radical Littré Quiroga Arenas objetó las demandas estudiantiles defendidas por Quevedo, señalando que quienes afirmaban que la germanización inspiraba todos los trabajos de la Escuela de Artes, no hacían más que manifestar su desconocimiento e ignorancia, pues a su juicio, "no hay pueblo alguno 
de la Tierra, que lleve ventaja a la nación alemana en esta materia de organización de sus escuelas industriales" (CD, Ss. 50a ordinaria, 1 de septiembre de 1931, p 1840) lo que efectivamente era innegable, pero que chocaba con el proyecto de desarrollo nacional vinculado al fomento de la industrialización. Junto a estos reclamos, los alumnos demandaban a la dirección de la Escuela una mayor promoción a la carrera académica, criticando al director Montero, pues al colocarse en una situación de aislamiento con respecto a los alumnos, los obligaba "a abandonar sus aspiraciones" (CD, Ss. 49a ordinaria, 31 de agosto de 1931, p. 1795). Esto explica que una de las demandas más sentidas de los estudiantes, fuera la entrega de la ejecución de la enseñanza industrial "a los exalumnos de la Escuela de Artes y Oficios" (CD, Ss. $50^{a}$ ordinaria, 1 de septiembre de 1931, p. 1841). Ante lo cual, el diputado Quiroga replicó que el cumplimiento de esta demanda era imposible, puesto que hacerlo constituiría una paradoja respecto del diagnóstico realizado por los mismos estudiantes, autodefinidos como fracasados en su formación profesional.

El conflicto entre los estudiantes y la dirección de la EAO llevó al Presidente Juan Esteban Montero a decretar en el año 31 el cierre temporal de la Escuela ${ }^{11}$. No obstante, y como toda situación de crisis, junto con la crítica se abrió un contexto de oportunidades favorable para el impulso de proyectos de reforma a la estructura administrativa y funciones de la Madre Escuela, que se incorporaron dentro de las demandas por cambiar el estatus de la educación técnica, lo que usualmente se planteó como una 'dignificación' de quienes estudiaban para cumplir labores de trabajo manual respecto de quienes lo hacían para incorporarse a la burocracia administrativa, éstos últimos concentrados en la Universidad de Chile. De este modo, el diputado Cardenio González ${ }^{12}$ explicitó su molestia frente a las medidas asumidas por el Gobierno señalando que:

11 Esto ocurrió en un contexto de permanentes convulsiones políticas que al año siguiente llevarían a la forzosa salida de Montero de la presidencia y al establecimiento de la efímera 'República Socialista' de Marmaduque Grove.

12 De militancia independiente, Cardenio González puede ser considerado a su vez un ejemplo de estudiante técnico y conocedor de la realidad estudiantil, en tanto estudió en escuelas técnicas chilenas y norteamericanas, desempeñando labores en la Empresa de Ferrocarriles del Estado, y siendo miembro de la Asociación de Ferrocarriles a la fecha de su desempeño como diputado. 
"mientras en la Universidad se cambia de Rector cada vez que el alumnado lo pide, mientras en la Universidad se pide que se modifique el Estatuto Universitario y se modifica, en los planteles inferiores, como la escuela de Artes y Oficios y la Escuela José Abelardo Núñez, lo que se hace en contestación a una petición del alumnado, es clausurar el establecimiento porque dichos alumnos son de una clase social distinta, en un 99 por ciento, a la de los estudiantes de la Universidad de Chile" (CD, Ss. 42a ordinaria, 24 de noviembre de 1931, p. 1598).

El argumento de que el origen de clase social de los estudiantes era el que determinó las drásticas medidas tomadas por la autoridad daba cuenta de que habían sectores políticos, con poder de decisión, para los cuales resolver los problemas y limitaciones del sistema de educación técnica no constituía una prioridad, demostrando desconfianza del sendero industrializador, que en los primeros años de la década de 1930 comenzaba paulatinamente a volverse hegemónico, pero tenían la resistencia de un gobierno para el cual, a pesar de que la EAO contaba con más de ochenta años de existencia, aún estimaba que "no era necesaria una modificación de sus programas y reglamentos internos" (CD, Ss. 42a ordinaria, 24 de noviembre de 1931, p. 1598). Del mismo modo, para Cardenio González la Escuela era "un verdadero claustro, un verdadero cuartel, sus alumnos no tienen ningún roce social con nadie" (CD, Ss. $42^{\mathrm{a}}$ ordinaria, 24 de noviembre de 1931, p. 1599), lo que hacía urgente su modernización y vínculo con la sociedad. En suma, la Escuela de Artes y Oficios se evaluaba como incapaz de cumplir con las necesidades que a la sazón tenía el país y con los proyectos de futuro tendientes a la industrialización. Esto último constituía el principal escollo para la implementación de reformas en la estructura económica nacional, como denuncia González, puesto que hacia 1931:

"nuestro país no es capaz todavía de producir un ingeniero mecánico, un ingeniero electricista, un metalurgista. Dentro de poco, vamos a poder elaborar fierro y acero en el país y no tenemos un técnico metalurgista" (CD, Ss. $42^{\mathrm{a}}$ ordinaria, 24 de noviembre de 1931, p. 1599). 
En consecuencia, la crisis de la Escuela levantó la opción de cuestionar su rol en la sociedad, en función de su vínculo con los nuevos proyectos de desarrollo nacional. Las opiniones esgrimidas por el mismo diputado González constituyen una buena síntesis de lo anterior, planteando que la EAO:

"necesita un remozamiento en sus programas, a fin de que vaya preparando en ella a los técnicos industriales si queremos hacer avanzar nuestras industrias, pues, de otro modo, creo que ningún gobierno podrá desarrollar un programa de industrialización sin disponer de un personal técnico suficiente y preparado que vaya a aportar sus conocimientos frente a las industrias, haciéndolas surgir" (CD, Ss. 42 a ordinaria, 24 de noviembre de 1931, p. 1599).

La evaluación de que la Escuela se encontraba desconectada de la realidad de la sociedad chilena y la incapacidad de generar cuadros técnicos que condujeran los procesos de industrialización, promovieron la demanda por fundar una institución de educación superior que corrigiera las deficiencias y complejizara las funciones de la EAO. Las discusiones en torno a la creación de una universidad industrial constituyeron una constante en el parlamento, encontrando una mayor resonancia hacia 1940, en el marco del homenaje al $91^{\circ}$ Aniversario de la fundación de la Escuela de Artes y Oficios. Las demandas fueron conducidas por el diputado del Partido Socialista César Godoy Urrutia, al señalar que los estudiantes de la EAO:

"desean que se lleve a la práctica una vieja aspiración y que ya está, puede decirse, a medio camino. Es la creación de lo que se llama el tercer grado de la enseñanza, para formar ingenieros industriales [...]. Veríamos con mucho agrado que, con motivo de cumplirse ahora el cuasi centenario de esta Escuela, institución gloriosa para la educación de Chile, se le diera forma a esta idea y, en definitiva, se procurara completar así la educación técnica y especialista que se propicia en la Escuela de Artes y Oficios" (CD, Ss. $15^{\mathrm{a}}$ ordinaria, 3 de julio de 1940, p. 1012). 
Esta demanda, compartida por el grueso de los parlamentarios, adquirió mayor capacidad organizativa tras la fundación de la Federación de Estudiantes Mineros e Industriales de Chile (FEMICH) ${ }^{13}$, en 1945, que aglutinó los anhelos provenientes desde los estudiantes de la EAO, la Escuela de Ingenieros Industriales (EII) y de las escuelas de minas a nivel nacional. Este objetivo de articular una institución de educación técnica de carácter superior se cumplió en abril de 1947, con la fundación de la Universidad Técnica del Estado por el gobierno del Presidente Gabriel González Videla (Muñoz et al, 1987 $)^{14}$. Sin embargo, la UTE entró en funcionamiento recién en 1952, demora que generó críticas por parte de algunos parlamentarios, expresadas durante las celebraciones del centenario de la EAO. Los diputados argumentaron que el desarrollo económico sustentado en el énfasis de las industrias nacionales se encontraba en una etapa decisiva, y necesariamente debía ser acompañada por la enseñanza industrial en el país. Francisco Javier Lira Merino, a nombre de "los diputados del Partido Agrario Laborista, junto con los diputados de la Falange Nacional" (CD, Ss. $14^{a}$ ordinaria, 6 de julio de 1949, p. 505), adhirieron al aniversario en momentos "en que el país comienza a caminar por una etapa de industrialización, que es cuando necesita de especialistas" (CD, Ss. 14ª ordinaria, 6 de julio de 1949, p. 505) Quien introdujo un énfasis distinto en las celebraciones de la fundación de la UTE fue el Ministro de Educación, Alejandro Ríos Valdivia. En su intervención en el hemiciclo sostuvo que en la coyuntura descrita se hacía imperioso el deber del Estado "de trazar nuevos rumbos a la educación pública, tratando de dar un desarrollo cada vez mayor a la enseñanza profesional o técnica, la que debe ser parte de la política económica general" (CD, Ss. 14ª ordinaria, 6 de julio de 1949, p. 501), Ríos Valdivia enfatizó en su argumentación que el gobierno diera definitivamente marcha a la UTE, por el proyecto de desarrollo nacional y por los anhelos de los estudiantes, afirmando que:

$13 \mathrm{La} \mathrm{FEMICH}$, tuvo influencia especialmente en los diputados de izquierda y, particularmente, en los comunistas, correligionarios de los principales dirigentes de la Federación.

14 La UTE se conformó con la unión de las principales escuelas politécnicas del país, ellas eran: las escuelas de minas de Copiapó, Antofagasta y La Serena; las escuelas industriales de Concepción y Valdivia, además de las EAO de Santiago y la EII. En 1948, a todas estas instituciones se sumaron la Escuela Industrial de Temuco y el Instituto Pedagógico Técnico. 
"Yo espero que el actual gobierno culminará su acción en beneficio del desarrollo industrial del país, enviando a la brevedad posible a esta Cámara el proyecto de ley que establece el Estatuto Orgánico de la Universidad Técnica del Estado. Esta es una aspiración sentida desde hace muchos años, que viene a culminar los anhelos de los que comienzan en la enseñanza profesional en el grado de oficiales para seguir al grado de técnicos y que quieren recibir el espaldarazo definitivo que les permita dirigir la industria de este país, obteniendo en la Universidad Técnica del Estado sus títulos de ingenieros en las diversas especialidades" (CD, Ss. 14 a ordinaria, 6 de julio de 1949, p. 501).

En 1952, con la dictación del Estatuto Orgánico se dio inicio a las actividades de la UTE, culminando así el proyecto de potenciar la formación de profesionales para el desarrollo nacional. La implementación del modelo ISI posibilitaba contar entonces con operarios nacionales calificados que, junto con aportar al desarrollo del proyecto de modernización económica, ayudarían a consolidar la tendencia a revertir la supremacía de los operarios extranjeros en la enseñanza y fábricas, denunciada veinte años atrás, pero que según el diputado Lira Merino, hacia fines de los 40 ya se podía apreciar cómo estos hombres,

"salidos de nuestras propias escuelas técnicas, como la Escuela de Artes y Oficios, han ido desplazando a los elementos extranjeros, a los cuales ha habido necesidad de recurrir en numerosas industrias nacionales. Y hemos podido comprobar que estos elementos nacionales han sido tanto o más eficientes que aquellos venidos de ultramar" (CD, Ss. 14 a ordinaria, 6 de julio de 1949 , p. 505).

A inicios de la década de 1950, los diputados de todos los sectores políticos realizaron positivas evaluaciones de la reforma al sistema de educación técnica y del logro de aquellas demandas que se habían coronado con la fundación de la UTE, lo que al mismo tiempo significaba para los parlamentarios un triunfo para la mano de obra chilena, definida como piedra angular del proyecto de 
desarrollo nacional. Sin embargo, a poco andar, estas auspiciosas evaluaciones se toparon con la crisis del modelo ISI, la que evidenció el carácter parcial de las transformaciones a la estructura económica nacional. En el proceso de industrialización se había optado por privilegiar elementos propios de la primera fase o 'etapa fácil' del proceso de modernización económica, caracterizada por el fomento a la industria liviana, pero sin 'dar el salto', hacia una segunda fase, complejizando este proceso mediante el fomento a la industria pesada y, principalmente, por medio de la ampliación del mercado interno a través de la modernización de las relaciones sociales de producción en el espacio rural, limitaciones estructurales que se expresaron con fuerza hacia mediados de los 50, determinando la crisis de la Industrialización Sustitutiva de Importaciones.

\section{Conclusiones: Una transformación parcial}

Las dos guerras mundiales, y especialmente la crisis de 1929, hicieron a los políticos nacionales sacar lecciones que modificaron parcialmente la estructura económica y social de Chile. La toma de conciencia de la vulnerabilidad derivada de la economía primario exportadora hizo que la estrategia de propender al desarrollo de las industrias nacionales progresivamente se hiciera hegemónica entre los parlamentarios de todas las tendencias, poniendo en el centro de las reformas el problema de la educación técnica como medio para corregir las deficiencias de mano de obra calificada. En este sentido, destaca también la rapidez inicial en la elaboración de propuestas demostrada por los parlamentarios, que surgieron prácticamente de forma paralela a las primeras expresiones de la crisis y emanaron fundamentalmente desde la Cámara de Diputados. Consideramos que esta capacidad de respuesta en términos propositivos da cuenta precisamente de la conciencia existente respecto de la vulnerabilidad de la economía chilena, pudiendo comprenderse como anticipos o resguardos de los dramáticos efectos que con la crisis económica arribaron a suelo nacional.

En cuanto a las propuestas de reforma al sistema de educación técnica vinculadas a la implementación del modelo ISI, pueden reconocerse propuestas provenientes de distintos sectores políticos, 
principalmente de los partidos con tendencia de izquierda, donde destaca el Comunista ${ }^{15}$, o de centro izquierda, especialmente el Demócrata y el Radical que, a pesar de sus matices, enfatizaron en el rol del Estado, ya sea como principal agente y promotor de ellas, como fue de la UMT y la UTE, o como simple facilitador de iniciativas que terminarían siendo privadas, como en el caso de la propuesta de fundación del Instituto de Perfeccionamiento Técnico. Así como todas las propuestas destacan el rol del Estado, las más elaboradas tienden a sustentarse ya sea en experiencias anteriores, como la UMT, o bien en un sistema administrativo preestablecido y de propiedad estatal. El mejor ejemplo de esto último, lo constituyó la creación de la UTE, en tanto sumatoria de diversas escuelas técnicas, industriales y de minas, aglutinadas en torno a la EII y a la EAO.

En el periodo estudiado, el consenso por la necesidad de reformar el sistema de educación técnica se mantuvo entre los parlamentarios a pesar de las distintas plataformas políticas de sus respectivos partidos. Del mismo modo, dicho consenso se aglutinó en torno a la idea de que las reformas económico-educacionales, al enmarcase dentro de un proyecto de desarrollo nacional, debían necesariamente incorporar la defensa de los obreros y estudiantes chilenos. Asimismo, las reformas al sistema educativo fueron concebidas, no solo como un medio para dotar a los futuros técnicos de conocimientos teóricos que les permitieran el mejoramiento material, sino que éstas se relacionaron estrechamente con la reforma de la sociedad y la moralización de la población. En consecuencia, las ideas de progreso nacional superaron el aspecto económico arribando hacia el educativo, concebido como espacio de resocialización de los sujetos. Por tanto, si el progreso material iba a parir en las industrias, las escuelas y la UTE darían vida al progreso social, normando las conductas e incorporando nuevos hábitos y valores en los sectores populares, principales demandantes de la educación técnica.

Por último, reafirmamos la existencia de un desfase entre las reformas educacionales y las económicas, cuya expresión no fue solo

15 Cabe destacar que en 1933 la Conferencia Nacional del PC Chile, aprobó la línea política de la Revolución Democrático Burguesa, que lo vinculó con sectores de centro e izquierda que compartían las medidas de industrialización nacional. 
temporal sino también en sus objetivos estratégicos, volviendo tardías o insuficientes las reformas al sistema educativo en la medida que sus limitaciones no se resolvieron, a pesar de la fundación de instituciones como la CORFO, del consenso político en torno a las reformas de la economía nacional orientadas a potenciar su industrialización, y de las transformaciones en el sistema de educación técnica de carácter industrial -conducidas principalmente por los gobiernos radicales- con el fin de alinearse y sostener este proceso. Así, los cambios pueden considerarse como profundos solo si se miran en el corto plazo, mas, una mirada detenida del proceso da cuenta de que esas reformas no alcanzaron espesor ni densidad en la medida que no se completaron o fueron incapaces de englobar al conjunto del sistema económico y de los actores sociales. Por tanto, las medidas económico-sociales y educacionales asumidas, dan cuenta de una modernización parcial, que hacia 1955 ya daba visos de agotamiento. En síntesis, los consensos iniciales, encontraron sus límites cuando la industrialización, tras superar la etapa 'fácil', debía pasar a un nivel de mayor complejización, contrapunto donde se enfrentaron los diversos proyectos de desarrollo nacional o de sociedad. De tal forma, utilizar a plenitud las potencialidades de la UTE requería de un proyecto modernizador distinto al aquí analizado, y que se hizo preponderante recién en el primer tercio de 1970.

\section{Referencias bibliográficas}

Aguirre Cerda P. (1933) El problema industrial, Santiago: Editorial Universidad de Chile.

Ahumada, J. (1973) En vez de la miseria, Santiago: Editorial Los Andes.

Alessandri, J. (1955) La verdadera situación económica y social de Chile en la actualidad, Santiago: Editorial Universitaria.

Arellano, J.P. (1985) Politicas sociales y desarrollo. Chile 1924-1984, Santiago: Editorial Alfabeta.

Aylwin, M.; Bascuñán, C.; Correa, S.; Gazmuri, C.; Serrano, S.; Tagle, M. (2002) Chile en el siglo XX, Santiago: Editorial Planeta, 2002, p. 115.

Cámara de Diputados (1931). Diario de Sesiones (Ss.) 8a ordinaria, p. 223.

Collier, S. y Sater, W.F. (2000) Historia de Chile 1804-1994, Cambridge: Editorial Cambridge. 
Devés, E. (2000) El pensamiento Latinoamericano en el siglo XX, Tomo I. Del Ariel de Rodó a la CEPAL (1900-1950), Buenos Aires: Editorial Biblos-Centro de Investigaciones Diego Barros Arana.

Devés, E. (2003) El pensamiento Latinoamericano en el siglo XX, Tomo II. Desde la CEPAL al Neoliberalismo (1950-1990), Buenos Aires: Editorial BiblosCentro de Investigaciones Diego Barros Arana.

Devés, E. (2004) El pensamiento Latinoamericano en el siglo XX, Tomo III. Las discusiones y las figuras de fin de siglo. Los años '90, Buenos Aires: Editorial Biblos-Centro de Investigaciones Diego Barros Arana.

Faúndez, J. (1992) Izquierdas y democracia en Chile. 1932-1973, Santiago: Editorial Bat.

Godoy, H. (2000) Estructura social de Chile, Santiago: Editorial Andros.

González Videla, G. (1975) Memorias. Santiago: Editorial Gabriela Mistral.

Jobet, J. C. (1955) Ensayo Crítico del desarrollo económico-social de Chile, Santiago: Editorial Universitaria.

Lagos, R. (1960) La concentración del poder económico en Chile, Santiago: Editorial del Pacífico.

Meller, P. (2007) Un siglo de economía política chilena (1890-1990), Santiago: Editorial Andrés Bello.

Milos, P. (2008) El Frente Popular en Chile: su configuración 1935-1938, Santiago: Editorial Lom.

Moulian, T. (2006) Fracturas: de Pedro Aguirre Cerda a Salvador Allende (19381973), Santiago: Editorial Lom.

Muñoz, J. G.; Norambuena C., Ortega L. y Pérez, R. (1987) La Universidad de Santiago de Chile: sobre sus orígenes y su desarrollo histórico, Santiago: Editorial USACH.

Muñoz, O. (1986), Chile y su industrialización, Santiago: Editorial Alfabeta.

Ortega, L. (2005) Chile en ruta al capitalismo. Cambio, euforia y depresión (18501880), Santiago: Editorial Dibam-Lom.

Ortega, L. (Coordinador) (1989) Corporación de Fomento de la Producción: 50 años de realizaciones 1939-1989, Santiago: Editorial Universidad de Santiago.

Pinto, A. (1959) Chile, un caso de desarrollo frustrado, Santiago: Editorial Universitaria.

Senado de la República. Diario de Sesiones (1920-1955). 
102 LOS DESAFÍOS DE LA INDUSTRIALIZACIÓN: DEBATES Y PROPUESTAS PARLAMENTARIAS EN TORNO A LA EDUCACIÓN TÉCNICA INDUSTRIAL. CHILE 1929-1952 - F. Rivera

Salazar, G. y Pinto, J. (1999) Historia contemporánea de Chile (vols. 1 y 2), Santiago: Editorial Lom.

Salazar, G. y Pinto, J. (2002) Historia contemporánea de Chile (vol. 3, 4 y 5), Santiago: Editorial Lom.

Recibido: 29/03/2011

Aceptado: 25/04/2011 